\title{
SUCCESSFUL EXTENSIVE MULTIORGAN RESECTION OF MALIGNANT DUODENAL TUMOR: A CASE REPORT
}

\author{
Ilva Spiridonova*, Armands Sīvin̄š **,*** \\ * Riga Stradins University, Riga, Latvia \\ ** Latvia Oncology Center, Riga East University Hospital, Riga, Latvia \\ *** Department of surgery, University of Latvia, Riga, Latvia
}

\section{Summary}

Duodenal adenocarcinoma is one of the rarest tumours of all gastrointestinal malignancies. Due to rarity and late symptoms, duodenal cancers are diagnosed in late stage, but overall survival rate is high. We present a case of locally advanced duodenal adenocarcinoma with successful surgical treatment including multiorgan resection.

Keywords: duodenal adenocarcinoma, Whipple procedure, duodenal bulb

\section{AIM OF THE DEMONSTRATION}

The aim of demonstration is to report about our experience with malignant duodenal tumour in order to emphasize the possibilities of modern oncologic surgery in the treatment of advanced tumours by performing multiorgan resections.

\section{CASE REPORT}

Planned hospitalisation of a 72-year-old male. Admitted due to periodic stabbing pain in the abdomen after eating, loss of appetite which resulted as $10 \mathrm{~kg}$ weight loss in 2 previous months, upon admission, anaemia was evident ( $\mathrm{Hb} 7.3 \mathrm{~g} / \mathrm{dL})$. His medical history included arterial hypertension, atherosclerosis and implantation of cardiac pacemaker due to atrioventricular block, grade I. Benign prostate hyperplasia was treated surgically 5 years before the current admission.

Upper endoscopy revealed a prepyloric deformation and a large semicircular ulcer in the anterior wall of duodenal bulb. Biopsy of the ulcer revealed chronic inflammation of low activity. Abdominal contrastenhanced computed tomography (CT) revealed inhomogeneous mass in the duodenal bulb and descending part. The mass measured 57.2 x 63.9 $\mathrm{mm}$ and seemed to invade the pancreatic head and transverse colon (Fig.1). All of previously done tests aided staging of the malign mass. Laboratory evaluation displayed elevated WBC count $\left(10.4 \times 10^{9} /\right.$ L), anaemia (after blood transfusion, Hb reached 10.3 $\mathrm{g} / \mathrm{dL}$ ) and thrombocytosis $\left(508 \times 10^{9} / \mathrm{L}\right)$. CEA and CA 19-9 revealed no elevations.

Patients case was presented to radiology council. It concluded that the tumor was operable, a decision was made to perform Whipple procedure, combined with right hemicolectomy, D2 and paraaortic lymph node dissection (6). Decision was based on clinical findings in upper endoscopy and contrast-enhanced CT scan (Fig.2). Operation included five anastomoses: pancreatojejunal, done in two layers, billiodigestive hepaticojejunal, done with separate sutures, gastroentero, entero-entero and ileotransversal anastomoses which was performed in 2 layers, with separated sutures. The surgery lasted $7 \mathrm{~h}$. During the surgery there were no major complications, blood loss was measured from 200 to $300 \mathrm{ml}$, blood transfusion was not needed. Two drains were added in the final phase of operation: subhepatically under the biliodigestive anastomosis and by the pancreatojejunal anastomosis. Evacuation of the drains was performed at the day of discharge, $18^{\text {th }}$ postoperative day. The whole postoperative period the patient received Sandostatin, to avoid development of pancreatic fistula. Pathohistological examination revealed adenocarcinoma of duodenum $\mathrm{pT}_{4} \mathrm{~N}_{1} \mathrm{M}_{0} \mathrm{~L}_{+} \mathrm{V}$ $\mathrm{R}_{0} \mathrm{G}_{2}$, stage III, KI-67 - 80\%. The tumor had invaded head of pancreas, transverse colon, transverse mesocolon, gall bladder and lymphatic vessels. Tumor was accompanied by necrosis and perineural growth. Pathologist extracted 9 lymphnodes, 2 with evidence of metastases.

The patient spent 5 days in ICU and subsequently transferred to surgical department for 13 days. Patient recovered with no further complications and was discharged from the hospital on the $18^{\text {th }}$ postoperative day in good general condition. Patient received adjuvant chemotherapy of FOLFOX6 for 4 cycles, according to colorectal cancer treatment guidelines (1). There were no major side-effects, patient was able to receive the whole medication course. Roughly three months after chemotherapy course patient developed toxic polyneuropathy which is a common side-effect for FOLFOX6 course. No metastases were observed during hospitalization and surveillance. Laboratory investigations and multiple contrast-enhanced CT scans were performed 5 and 8 months later, with no evidence of recurrent malignancy. 


\section{DISCUSSION}

In RAKUS hospital a hospital wide investigation was done to find out the correlation between how many lymph nodes were resected and which pathologist performed it. A correlation was found between the resected lymph node count in the specimen and the pathologist who performed it. This patient received D2 lymphadenectomy with paraaortic lymph node dissection, but unfortunately in the pathohistological report there were only 9 lymph nodes of which 2 were with metastases, it was not specified which lymph node groups were positive.

Primary adenocarcinoma of duodenum is a rare malignancy accounting for less than $1 \%$ of all gastrointestinal cancers (2). Given the rarity of the disease, there is limited guidance for treatment; however, it has a favourable outcome. Majority of duodenal adenocarcinomas arise in the second portion of duodenum. Diagnosis is difficult and often delayed. Symptoms are nonspecific and include abdominal pain, nausea, vomiting, weakness. Such symptoms as jaundice, anaemia, gastrointestinal obstruction is associated with advanced disease (2). At the moment of diagnosis, $24-39 \%$ of patients demonstrate cancer stage III (3).

The goal of surgical treatment is margin negative resection (R0) of a lesion and local lymphadenectomy. Recommended method of treatment duodenal invasive tumours is pancreaticoduodenectomy (PD). In case of a tumour located outside the ampulla of Vater and not infiltrating the surrounding tissues, segmental resection (SR) of duodenum can be performed (1). So far, no well-established chemotherapy has been identified.

Small bowel adenocarcinomas are treated similar to colorectal adenocarcinomas with oxaliplatin based chemotherapy (4). In the majority of studies, 5fluorouracil (5-FU) was used as the main agent or combined with platinum-based agents (oxaliplatin, cisplatin and carboplatin), and resulting in a better response to treatment. In a group of patients with a high risk of disease recurrence (more than 10\% of resected lymph nodes were metastatic) adjuvant treatment improved the overall survival (OS), but not the disease-free survival (DFS) (5). Recently published data indicates a statistically significant improvement in OS in stage III patients, who received adjuvant chemotherapy (42.4 vs. 26.1 months) (3). The role of adjuvant radiotherapy in the treatment of duodenal adenocarcinoma is not well defined. A retrospective study of 32 patients from Duke University Medical Center was able to show modest improvement in local control $(70 \%$ vs $49 \%)$ with adjuvant chemoradiation therapy (5).

Duodenal adenocarcinoma represents aggressive cancer, but in patients with resectable tumor, longterm outcomes are better than in cases of other periampullary malignancies. In a retrospective study of 122 patients who underwent PD for duodenal adenocarcinoma over a 22-year period at MSKCC, ten-year OS was $41 \%$. Negative prognostic factors are age $>65$, pT3-T4, positive regional lymph nodes, poorly differentiated tumour, positive resection margins (3). In conclusion, a multidisciplinary approach with extensive radical surgery followed by adjuvant chemotherapy leads to improved survival and quality of life.

\section{Conflict of interest: None}

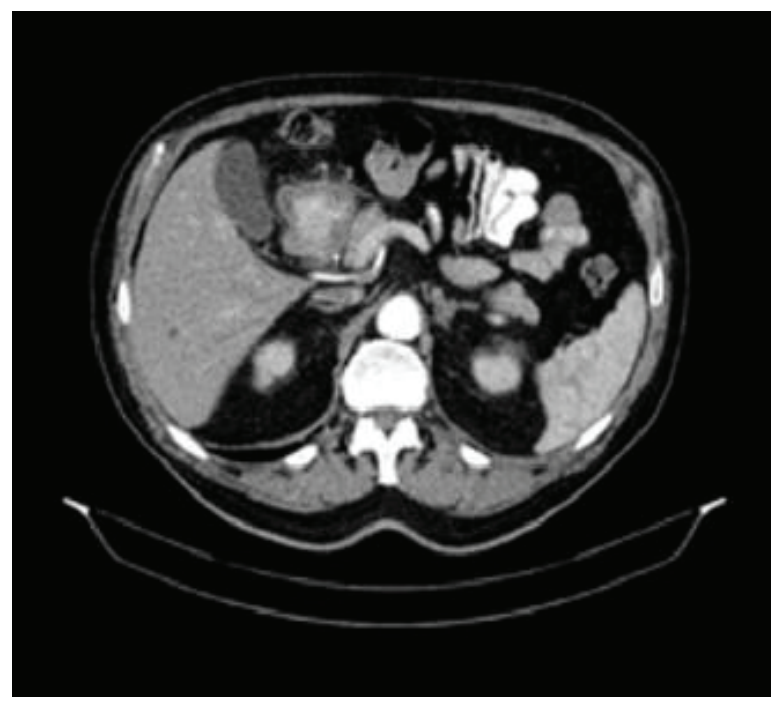

Fig.1. CT-scan before surgery shows inhomogeneous contrast enhancing mass in duodenum

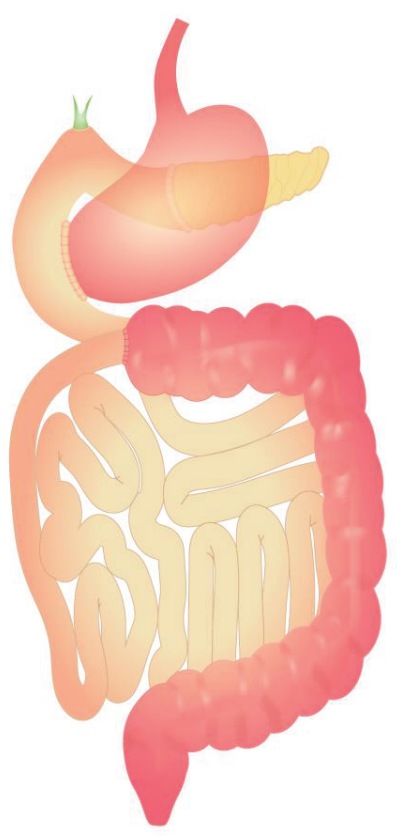

Fig.2. Pancreatoduodenal resection m. Whipple with right hemicolectomy. Author: Arvita Klaviņa 


\section{REFERENCES}

1. Aparicio $\mathrm{T}$ et al. Small bowel adenocarcinoma: epidemiology, risk factors, diagnosis and treatment. Dig Liver Dis. 2014;46(2):97-104

2. CloydJMetal.Duodenaladenocarcinoma:Advances in diagnosis and surgical management. World J Gastrointest Surg. 2016;8(3):212-221

3. Ecker BL et al. Efficacy of adjuvant chemotherapy for small bowel adenocarcinoma: A propensity score-matched analysis. Cancer. 2016;122: 693-701

4. Hochsten HS et al. Improved time to treatment failure with an intermittent oxaliplatin strategy: results of CONcePT. Ann Oncol. 2014;25(6): 1172-8

5. Lech $\mathrm{G}$ et al. Primary small bowel adenocarcinoma: current view on clinical features, risk and prognostic factors, treatment and outcome. Scand J Gastroenterol. 2017;52(11):1 194-1202

6. Zhang CD et al. Modified vs. standard D2 lymphadenectomy in distal subtotal gastrectomy for locally advanced gastric cancer patients under 70 years of age. Oncol Lett. 2018;15(1):375-385

\section{Address:}

Ilva Spiridonova

E-mail: spiridonovailva@gmail.com

Dzirciema 16, Riga, LV-1007 